\title{
Comparison of weight-for-height and mid-upper arm circumference (MUAC) in a therapeutic feeding programme in South Sudan: is MUAC alone a sufficient criterion for admission of children at high risk of mortality?
}

\author{
Emmanuel Grellety ${ }^{1, *}$, L Kendall Krause ${ }^{2}$, Manal Shams Eldin ${ }^{3}$, Klaudia Porten ${ }^{1}$ and \\ Sheila Isanaka ${ }^{1}$ \\ ${ }^{1}$ Epicentre, 8 rue Saint Sabin, 75011 Paris, France: ${ }^{2}$ Montevia Health, Denver, CO, USA: ${ }^{3}$ Médecins Sans \\ Frontières, Paris, France
}

Submitted 18 June 2014: Final revision received 26 January 2015: Accepted 3 February 2015: First published online 25 March 2015

\begin{abstract}
Objective: The present study was performed to describe the operational implications of using mid-upper arm circumference (MUAC) as a single admission criterion for treatment of severe acute malnutrition in South Sudan.

Design: We performed a retrospective analysis of routine programme data of children with severe acute malnutrition aged 6-59 months admitted to a therapeutic feeding programme using weight-for-height $Z$-score (WHZ) and/or MUAC. To understand the implications of using MUAC as a single admission criterion, we compared patient characteristics and treatment outcomes for children admitted with MUAC $<115 \mathrm{~mm}$ (irrespective of WHZ) $v$. children admitted with WHZ $<-3$ and MUAC $\geq 115 \mathrm{~mm}$.

Results: Of 2205 children included for analysis, 719 (32.6\%) were admitted to the programme with MUAC $<115 \mathrm{~mm}$ and 1486 (67.4\%) with $\mathrm{WHZ}<-3$ and MUAC $\geq 115 \mathrm{~mm}$. Children who would have been admitted using a single MUAC $<115 \mathrm{~mm}$ criterion were more severely malnourished and more likely to be female and younger. Compared with children admitted with $\mathrm{WHZ}<-3$ and MUAC $\geq 115 \mathrm{~mm}$, children who would have been admitted using MUAC $<115 \mathrm{~mm}$ were less likely to recover ( $54 \% v .69 \%)$ and had higher risk of death ( $4 \% v .1 \%)$, but responded to treatment with greater weight and MUAC gains. MUAC $<115 \mathrm{~mm}$ would have failed to identify $33 \%$ of deaths, while $98 \%$ were identified by WHZ $<-3$ alone and $100 \%$ by MUAC $<130 \mathrm{~mm}$.

Conclusions: The study shows that MUAC $<115 \mathrm{~mm}$ identified more severely malnourished children with a higher risk of mortality but failed to identify a third of the children who died. Admission criteria for therapeutic feeding should be adapted to the programmatic context with consideration for both operational and public health implications.
\end{abstract}

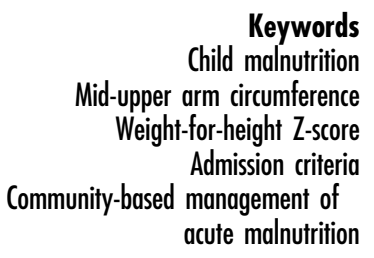

Acute malnutrition represents a major cause of childhood morbidity and mortality worldwide. The number of children under the age of 5 years with severe acute malnutrition (SAM) at any time is currently estimated from prevalence data to be nearly 19 million, with the burden or number of incident cases occurring each year presumably higher $^{(1)}$. SAM contributes to over a million child deaths annually, as children with SAM are estimated to have an approximately ninefold increased risk of death compared with well-nourished children ${ }^{(2,3)}$.

Traditionally, treatment for SAM was conducted exclusively in in-patient settings, an approach that was both costly and limited access to, and impact of, such programmes.
In 2007, a new model for the community-based management of acute malnutrition (CMAM) was endorsed by the WHO, UNICEF, World Food Programme and the UN System Standing Committee on Nutrition, in which children with uncomplicated cases of SAM and appetite could be treated on an out-patient basis with the provision of ready-to-use therapeutic foods and weekly or biweekly follow-up ${ }^{(3)}$. Increasing evidence and operational experience have demonstrated that community-based treatment of uncomplicated SAM is effective ${ }^{(4-7)}$ and cost-effective ${ }^{(8,9)}$.

Current guidelines recommend admission to therapeutic feeding if a child aged 6-59 months meets any one of the following three criteria: (i) weight-for-height $Z$-score 
(WHZ) of $<-3$ using the 2006 WHO Growth Standards; (ii) mid-upper arm circumference (MUAC) $<115 \mathrm{~mm}$; or (iii) presence of bilateral oedema ${ }^{(10)}$. Following increasing experience with CMAM and a focus on simplifying and scaling up therapeutic feeding, the possibility of using MUAC as the sole anthropometric criterion for admission to therapeutic feeding has been raised. Arguments in support of a broader use of MUAC include the simplicity of measurement, potential for improved coverage based on ease of implementation and low cost. In addition, MUAC has been found to be more sensitive at high specificity levels than WHZ in predicting mortality among children $^{(11-13)}$. Transition to a single MUAC criterion for admission to therapeutic feeding, however, is complicated by the fact that MUAC and WHZ are known to select different children at risk for acute malnutrition ${ }^{(14)}$, and operational experience from programmes using MUAC as a sole admission criterion remains limited.

The objective of the present study was to better understand the operational implications of using MUAC as a single admission criterion for therapeutic feeding. Using routine programme data from a Médecins Sans Frontières (MSF) therapeutic feeding programme (TFP) in Aweil, Northern Bahr El Ghazal, South Sudan, we compared population characteristics and treatment outcomes, including mortality, of children who would have been admitted for therapeutic feeding using MUAC $<115 \mathrm{~mm}$ alone $v$. children admitted for therapeutic feeding under the current $\mathrm{WHZ}<-3$ and/or MUAC $<115 \mathrm{~mm}$ criteria but who would have been excluded from therapeutic feeding if MUAC $<115 \mathrm{~mm}$ alone was used as a single anthropometric admission criterion.

\section{Methods}

\section{Programme description and study population}

South Sudan has one of the highest rates of malnutrition globally; the rate of acute malnutrition is approximately twice that seen in other countries in sub-Saharan Africa ${ }^{(15)}$. In August 2008, MSF opened a TFP in Aweil, Northern Bahr El Ghazal, a region characterized by historically high rates of SAM. The programme primarily served a Nilotic population ( $75 \%$ Dinka and $25 \%$ Luo ethnic groups), who are typically tall and slim. In such populations there has been discussion surrounding the suitability of WHZ to diagnose and monitor treatment response among children with $\operatorname{SAM}^{(16,17)}$.

Children aged 6-59 months were screened for malnutrition using weight, height (or length for children $<87 \mathrm{~cm}$ ), MUAC (for children with height $>65 \mathrm{~cm}$ ) and the presence of bilateral oedema; measurements were taken in accordance with WHO standard techniques ${ }^{(18)}$. Those children meeting at least one of two anthropometric criteria - WHZ $<-3$ or MUAC $<115 \mathrm{~mm}$ - and presenting with appetite and no medical complications were eligible for outpatient treatment, where home rations of ready-touse therapeutic food (Plumpy'nut ${ }^{\circledR}$, approximately 837 $\mathrm{kJ} / \mathrm{kg}$ body weight (BW) per d (200 kcal/kg BW per d)) were provided and weekly follow-up was conducted with physical examination, anthropometric assessment and distribution of the next therapeutic ration until discharge. Children were referred to in-patient treatment for deterioration of clinical status, including poor appetite, increasing or new oedema, or weight loss or lack of weight gain for three consecutive visits. Children were discharged as 'recovered' if they achieved WHZ $\geq-2$ and MUAC $>115 \mathrm{~mm}$ at two consecutive visits, were free of oedema for $>7 \mathrm{~d}$, and were clinically well for at least two consecutive follow-up visits.

The study population for the present analysis included all children aged 6-59 months admitted to out-patient care in the MSF TFP in Aweil, South Sudan with WHZ $<-3$ or MUAC $<115 \mathrm{~mm}$ from January 2010 to December 2010. Children with oedema were excluded from the analysis as the assessment of weight in the presence of oedema is not informative.

\section{Statistical analysis}

We performed a retrospective analysis of routine programme data of children aged 6-59 months admitted to the MSF outpatient TFP in Aweil, South Sudan in 2010. To describe the potential implications of using MUAC as the single anthropometric admission criterion in this setting, we compared patient characteristics and treatment outcomes between two groups in the 2010 programme population: (i) children admitted to the programme with MUAC $<115 \mathrm{~mm}$, regardless of WHZ (referred to as the 'MUAC+' group), to represent those children who would have been included for treatment if using MUAC as the single anthropometric admission criterion; $v$. (ii) children admitted with $\mathrm{WHZ}<-3$ and MUAC $\geq 115 \mathrm{~mm}$ (referred to as the 'MUAC-/WHZ+' group), to represent children potentially excluded from treatment when using MUAC as the single anthropometric admission criterion.

Treatment outcomes were defined for all children as follows.

1. Death: death from any cause during follow-up.

2. Default: children who failed to appear for two or more consecutive follow-up visits.

3. Transfer: children for whom out-patient management was deemed to be insufficient, including children with chronic illness, deterioration in clinical status, increasing or new oedema, or weight loss or failure to gain weight for at least three consecutive visits.

4. Recovered: children with $\mathrm{WHZ} \geq-2$ and MUAC $>115 \mathrm{~mm}$ at two consecutive visits and who were free of oedema for $>7 \mathrm{~d}$.

To compare baseline characteristics between the groups, the $\chi^{2}$ test was used to compare proportions, including age and sex, and a $t$ test was used to compare continuous variables, including nutritional status at 
admission. Binomial regression, adjusting for age and sex, was used to compare the risk of each treatment outcome between groups. For children classified as 'recovered', length of stay, change in MUAC and change in WHZ were calculated and compared between groups using linear regression, adjusting for age and sex. The level of significance was set at $P \leq 0 \cdot 05$. All data were analysed using R software version $2 \cdot 9 \cdot 2^{(19)}$. The data analyses were exempted from review by the MSF Ethical Review Board given the use of de-identified, routine programme data.

\section{Results}

\section{Characteristics of the study population}

A total of 2601 records were available for children admitted for out-patient treatment of uncomplicated SAM in 2010. For the present analysis, 396 (15.2\%) records were excluded: 107 ( $4 \cdot 1 \%$ ) for failing for meet stated programme admission criteria, sixty-five (2.5\%) for oedema on admission and 224 (8.6\%) for missing data on age, sex or anthropometry. Of the 2205 remaining eligible records, median age was 14 months (interquartile range $=10-24$ months) and 53\% were male. In the overall cohort, mean MUAC at admission was 117.5 (SD 9.1) $\mathrm{mm}$ and mean WHZ was -3.9 (SD 0.7; Table 1). Of the 2205 children included for analysis, 698 were admitted with both MUAC $<115 \mathrm{~mm}$ and $\mathrm{WHZ} \leq-3(31.6 \%$; MUAC $+/$ WHZ + ), twenty-one were admitted based on MUAC alone (1.0\%; MUAC+/WHZ-) and 1486 were admitted based on WHZ alone (67.4\%; MUAC-/WHZ +).

To better understand the programmatic implications for admission based on MUAC $<115 \mathrm{~mm}$ alone, we described and compared treatment outcomes of two groups: MUAC + $(n$ 719) $v$. MUAC $-/ \mathrm{WHZ}+(n$ 1486). Children admitted using MUAC as a single anthropometric admission criterion $(\mathrm{MUAC}+)$ were more likely to be female
(55\% v. 43\%; $P<0.0001)$ and younger (59\% v. $45 \%$ less than 12 months of age) than children who would not have been admitted with the single MUAC $<115 \mathrm{~mm}$ criterion (MUAC-/WHZ+). Children in the MUAC+ group had significantly lower MUAC $(107 \cdot 3 \mathrm{~mm} v$. $122.5 \mathrm{~mm} ; P<0.0001)$ and WHZ $(-4.3 v .-3.8 ; P<0.0001)$ on admission, compared with children in the MUAC-/ WHZ + group.

\section{Treatment outcomes}

Children in the MUAC + group were less likely to recover (54\%v. 69\%; $P<0.0001)$ and experienced a higher risk of death $(4 \%$ v. $1 \% ; P<0.0001)$ and transfer to in-patient care $(12 \% v .7 \% ; P<0.0001)$ compared with the MUAC -/ $\mathrm{WHZ}+$ group. In both groups, the risk of default was higher than the SPHERE standard of $<15 \%{ }^{(20)}$, with the risk being significantly higher among the MUAC + group compared with the MUAC-/WHZ + group (30\% v. $23 \%$; $P<0.0001$; Table 2). Among recovered children, treatment response was more favourable among the MUAC + group than the MUAC $-/ \mathrm{WHZ}+$ group: the MUAC + group had a greater mean weight gain $(6 \cdot 01 \mathrm{~g} / \mathrm{kg} \mathrm{BW}$ per $\mathrm{d} v \cdot 5 \cdot 12 \mathrm{~g} / \mathrm{kg}$ BW per $\mathrm{d} ; P<0.0001)$ and greater mean MUAC gain $(0.45 \mathrm{~mm} / \mathrm{d} v \cdot 0.25 \mathrm{~mm} / \mathrm{d} ; P<0.0001)$. Mean length of treatment was approximately 51 (SD 29.01) d overall and did not differ significantly between groups.

A total of forty deaths ( $2 \%$ ) were recorded in the study population, of which twenty-seven $(67.5 \%)$ were seen in the MUAC + group and thirteen (32.5\%) in the MUAC-/ $\mathrm{WHZ}+$ group (Fig. 1(a)). Adjusting for age and sex, the risk of death was over four times higher among children in the MUAC+ group compared with the MUAC-/WHZ+ group (risk ratio $=4 \cdot 02 ; 95 \% \mathrm{CI} 2 \cdot 10,8 \cdot 08$ ). Figure $1(\mathrm{~b})$ indicates the distribution of both MUAC and WHZ on admission for recorded deaths, showing that MUAC $<115 \mathrm{~mm}$ as the single anthropometric admission criterion

Table 1 Patient characteristics by admission criteria, children aged 6-59 months, Aweil, South Sudan, January-December 2010

\begin{tabular}{|c|c|c|c|c|c|c|c|}
\hline & \multicolumn{2}{|c|}{ All $(n$ 2205) } & \multicolumn{2}{|c|}{ MUAC + $(n 719)$} & \multicolumn{2}{|c|}{ MUAC- $/ \mathrm{WHZ}+(n$ 1486) } & \multirow[b]{2}{*}{$P$ value* } \\
\hline & $\%$ & $n$ & $\%$ & $n$ & $\%$ & $n$ & \\
\hline \multicolumn{8}{|l|}{ Sex } \\
\hline Male & 53 & 1170 & 45 & 327 & 57 & 843 & \multirow[t]{2}{*}{$<0.0001$} \\
\hline Female & 47 & 1035 & 55 & 392 & 43 & 643 & \\
\hline \multicolumn{8}{|l|}{ Age (months) } \\
\hline $6-12$ & 49 & 1086 & 59 & 418 & 45 & 668 & \multirow[t]{3}{*}{$<0.0001$} \\
\hline $13-24$ & 37 & 809 & 34 & 245 & 38 & 564 & \\
\hline \multirow[t]{2}{*}{$25-59$} & 14 & 310 & 7 & 56 & 17 & 254 & \\
\hline & Mean & SD & Mean & SD & Mean & SD & $P$ value* \\
\hline \multicolumn{8}{|c|}{ Nutritional status at admission } \\
\hline MUAC (mm) & 117.5 & $9 \cdot 1$ & $107 \cdot 3$ & $7 \cdot 0$ & $122 \cdot 5$ & $5 \cdot 0$ & $<0.0001$ \\
\hline WHZ & -3.9 & 0.7 & $-4 \cdot 3$ & 0.9 & $-3 \cdot 8$ & 0.5 & $<0.0001$ \\
\hline
\end{tabular}


Table 2 Treatment outcomes by admission criteria, children aged 6-59 months, Aweil, South Sudan, January-December 2010

\begin{tabular}{|c|c|c|c|c|c|c|c|c|c|}
\hline & \multicolumn{2}{|c|}{ All (n 2205) } & \multicolumn{2}{|c|}{ MUAC + $(n 719)$} & \multicolumn{2}{|c|}{ MUAC- $/ \mathrm{WHZ}+(n$ 1486) } & \multicolumn{3}{|c|}{ Reference: MUAC-MHZ+ } \\
\hline & $\%$ & $n$ & $\%$ & $n$ & $\%$ & $n$ & RR & $95 \% \mathrm{Cl}$ & $P$ value* \\
\hline \multicolumn{10}{|l|}{ Outcome } \\
\hline Death & 2 & 40 & 4 & 27 & 1 & 13 & 4.02 & $2 \cdot 10,8.08$ & $<0.0001$ \\
\hline Default & 25 & 564 & 30 & 218 & 23 & 346 & 1.36 & $1.17,1.58$ & $<0.0001$ \\
\hline Transfer & 9 & 190 & 12 & 89 & 7 & 101 & 1.87 & $1.42,2.47$ & $<0.0001$ \\
\hline \multirow[t]{2}{*}{ Recovered } & 64 & 1411 & 54 & 385 & 69 & 1026 & 0.77 & $0.71,0.83$ & $<0.0001$ \\
\hline & Mean & SD & Mean & SD & Mean & SD & Mean difference & $95 \% \mathrm{Cl}$ & $P$ value* \\
\hline \multicolumn{10}{|l|}{ Response for children recovered } \\
\hline Length of stay $(d)$ & 51.03 & $29 \cdot 01$ & 51.57 & 28.94 & $50 \cdot 82$ & $29 \cdot 04$ & 0.82 & $0.00,4.32$ & 0.647 \\
\hline Weight gain (g/kg BW per d) & $5 \cdot 36$ & $3 \cdot 10$ & 6.01 & 3.31 & $5 \cdot 12$ & 2.99 & 0.86 & $0.49,1.23$ & $<0.0001$ \\
\hline MUAC gain $(\mathrm{mm} / \mathrm{d})$ & 0.30 & 0.29 & 0.45 & 0.28 & 0.25 & 0.27 & 0.22 & $0.18,0.26$ & $<0.0001$ \\
\hline
\end{tabular}

MUAC, mid-upper arm circumference; WHZ, weight-for-height Z-score; RR, relative risk; BW, body weight.

MUAC+: includes children admitted based on single MUAC $<115 \mathrm{~mm}$ criterion, regardless of WHZ.

MUAC- $M H Z+$ : includes children currently admitted but excluded from treatment using a single $M U A C<115 \mathrm{~mm}$ admission criterion (WHZ $<-3$ and

$M U A C \geq 115 \mathrm{~mm}$.

${ }^{*}$ Proportions compared using binomial regression and continuous measures using linear regression; all models are adjusted for age and sex.

(a)

\begin{tabular}{|c|c|c|c|}
\hline & $\mathrm{WHZ}<-3$ & $W H Z \geq-3$ & \\
\hline \multirow[b]{2}{*}{$M U A C<115 \mathrm{~mm}$} & $(n 698)$ & $(n 21)$ & MUAC+ $(n 719)$ \\
\hline & 26 & 1 & Deaths: 27 (67.5\%) \\
\hline & $(n 1486)$ & & MUAC-/WHZ+ $(n$ 1486) \\
\hline MUAC $\geq 115 \mathrm{~mm}$ & 13 & & Deaths: $13(32.5 \%)$ \\
\hline
\end{tabular}

Total deaths: 40 (N2205)

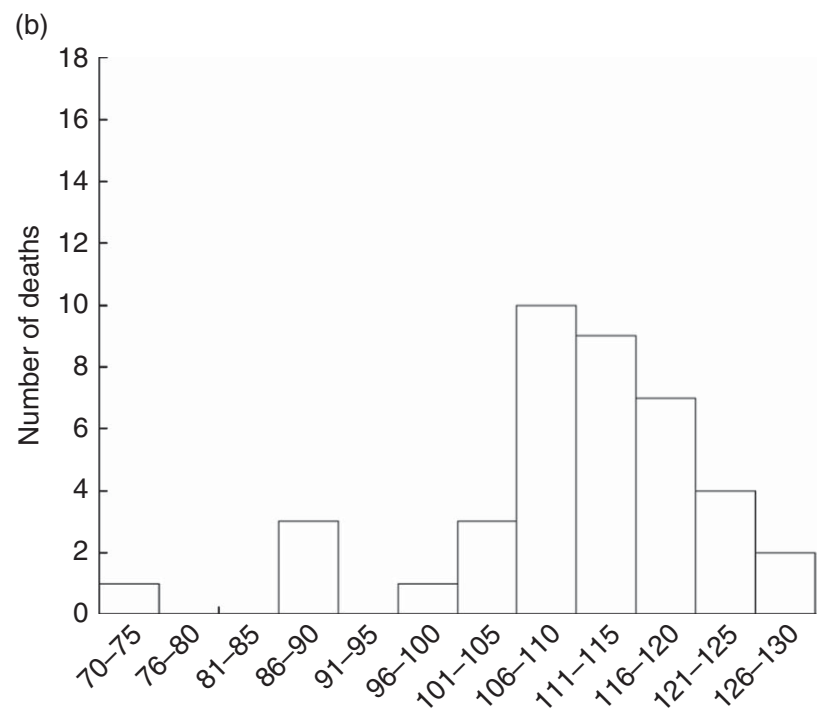

MUAC at admission ( $\mathrm{mm})$

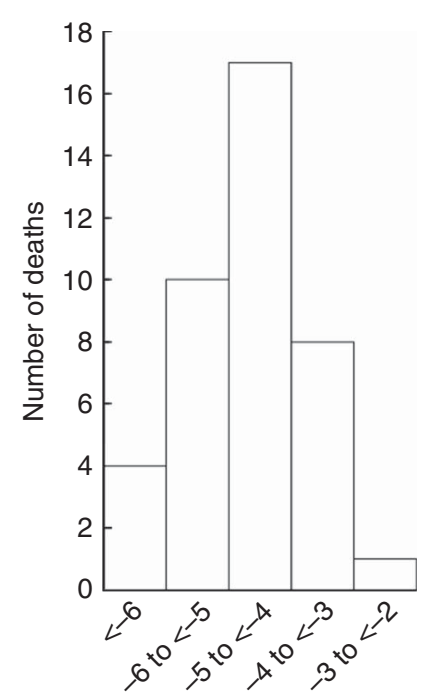

WHZ at admission

Fig. 1 (a) Death as outcome by anthropometric criteria on admission and (b) distribution of deaths in the study population by MUAC and WHZ upon admission, children aged 6-59 months, Aweil, South Sudan, January-December 2010 (MUAC, mid-upper arm circumference; WHZ, weight-for-height Z-score) 
would have missed $33 \%$ of deaths in the cohort. MUAC $<125 \mathrm{~mm}$ as the single anthropometric admission criterion would have captured all but two deaths in the cohort and all deaths would have been captured using MUAC $<130 \mathrm{~mm}$.

\section{Discussion}

We analysed data from an MSF TFP to describe patient characteristics and treatment outcomes of children who would have been admitted for out-patient treatment using MUAC $<115 \mathrm{~mm}$ as a single admission criterion $v$. those who would have been excluded using MUAC $<115 \mathrm{~mm}$ as a single admission criterion. We found that children who would have been admitted with a single MUAC $<115 \mathrm{~mm}$ criterion were more likely to be female and of younger age. These children were more severely malnourished, as indicated by lower MUAC and lower WHZ on admission. They were also at greater risk of default and death, but responded well to treatment in terms of weight and MUAC gain.

The MSF TFP in Aweil, South Sudan was opened in response to the high risk of SAM and other complicating factors in this setting. Some experience suggests, however, that WHZ may not be well suited for the diagnosis and follow-up of children with tall, slim body shapes, characteristic of some Nilotic populations. MSF programme experience in Aweil noted some difficulty of children admitted using WHZ criterion to meet discharge criteria for nutritional recovery, which generated interest in the possibility to adapt programme admission criteria to a single MUAC-based measure.

More broadly, MUAC has been proposed as a single admission criterion for several reasons, including its simplicity of use and correlation with increased risk of death. It is recognized that MUAC is more easily implemented than WHZ in field-based settings and facilitates community-based screening. Myatt et al. reviewed various indicators for case detection in the context of $\mathrm{SAM}^{(21)}$. The authors scored various indicators on the basis of a set of properties (including simplicity, acceptability, cost, objectivity, independence of age, precision/reliability, accuracy, sensitivity, specificity and predictive value) and concluded MUAC $<110 \mathrm{~mm}$ or the presence of bipedal oedema was the most appropriate screening criterion.

Despite support for broader use of MUAC as a single admission criterion, programmatic experience with MUAC-based admissions is limited. Few reports have been published to share experience from programmes admitting children with SAM to therapeutic feeding using MUAC only ${ }^{(20,22,23)}$. In these studies, treatment outcomes, including mortality, were similar to those recorded in our study. A study of the effect of a supplemental food programme in Guinea-Bissau found a mortality of $1 \%{ }^{(23)}$. Defourny et al. evaluated the use of MUAC $<110 \mathrm{~mm}$ as an admission criterion for a TFP in Niger ${ }^{(22)}$. The programme reported risks of death, default and non-response as $1.8 \%, 4.7 \%$ and $1.1 \%$, respectively. Average weight gain was $5 \cdot 1 \mathrm{~g} / \mathrm{kg}$ BW per $\mathrm{d}$ and average length of treatment was $44.4 \mathrm{~d}$, similar to outcomes seen in our programme (weight gain of $5.12 \mathrm{~g} / \mathrm{kg} \mathrm{BW}$ per $\mathrm{d}$ and median length of treatment of $50 \mathrm{~d}$ ).

The decision regarding the use of WHZ $v$. MUAC for admission to therapeutic feeding is complicated by the fact that each measure may select different children for treatment. Depending on the setting and geographic location, 40-90\% of children are not classified as severely malnourished based on both criteria ${ }^{(11,17,24-26)}$. Existing literature suggests that MUAC-based programmes tend to identify significantly more girls and younger children than those identified by $\mathrm{WHZ}^{(11)}$. Receiver-operating characteristic curves have also demonstrated that MUAC may identify children at a higher risk of mortality ${ }^{(18)}$. Our data confirm these findings.

We found that a MUAC cut-off of $<115 \mathrm{~mm}$ would have excluded $33 \%$ of the children who died in the programme, whereas a WHZ cut-off of $<-3$ identified almost all (98\%) children who died. This finding is in contrast with community studies in which low WHZ is less predictive of mortality than low MUAC ${ }^{(13,27)}$. The greater sensitivity of $\mathrm{WHZ}<-3$ to identify nearly all deaths observed in the present study population may be specific to the study context, which as a programmatic cohort represents a sub-sample of the general community that presented for and received treatment, and is drawn from a Nilotic population whose tall and slim body shape is associated with low WHZ. Nearly all children included in the present analysis were identified by low WHZ (only $1 \%$ of children were admitted with $\mathrm{WHZ} \geq-3$ ) and as a result, WHZ may be expected to more completely capture deaths here. As suggested by a previous analysis also carried out in a selected sample of treated children that aimed to compare the accuracy of WHZ and MUAC to predict death in a large nutritional programme in Niger $^{(28)}$, predicting death among children admitted to treatment is less useful than identifying which children in the community would benefit most (e.g. experience the greatest reduction in the risk of mortality) from treatment. Such comparison of mortality according to anthropometric criterion (e.g. WHZ $v$. MUAC $<115 \mathrm{~mm} v$. MUAC $<125 \mathrm{~mm}$ ) to determine which has superior accuracy and efficiency to identify children at risk of death in the absence of treatment would, however, be unethical. The present analysis should be viewed as a step to help to address this question in South Sudan, but cannot be used to draw firm conclusions.

While MUAC $<115 \mathrm{~mm}$ did not identify the majority of deaths in this setting, our results highlight how an upward adjustment of the MUAC admission threshold to MUAC $<125 \mathrm{~mm}$ could be applied to identify nearly all deaths (e.g. 95\%) in this setting. Any such upward adjustment would yield a more sensitive, but less specific, 
criterion for admission and consequently have important programmatic implications, including increased workload and costs. MSF experience in Burkina Faso in 2007-2009, where children were admitted to TFP with MUAC $\leq 118 \mathrm{~mm}$ or oedema, suggests children with MUAC of $116-118 \mathrm{~mm}$ benefited from treatment, as evidenced by rates of weight gain similar to those typically seen in $\mathrm{TFP}^{(20)}$. The authors conclude that the despite the 'programmatic price' of increased caseloads, a more sensitive MUAC cut-off may still be appropriate. The extent to which an upward adjustment in the MUAC admission threshold to MUAC $<125 \mathrm{~mm}$ would increase programme size and costs cannot be derived from the present data and would depend on the patient population (including age) and other operational factors, and therefore would require evaluation across various contexts. Additional experience with the use of MUAC for admission, monitoring and discharge is also needed. To date, few studies present information of children with SAM who were identified, monitored and discharged by weight and MUAC ${ }^{(11,29,30)}$.

Several limitations of the present analysis warrant mention. We note that the study population represents a selected sample of the general population (i.e. children admitted to the MSF TFP). Therefore, the results are not representative of the population at large and do not allow a clear conclusion about the relevance of using WHZ in addition to MUAC to select those children who have the highest risk of death in the community. Additional limitations of the analysis include the retrospective data collection, a risk of programme default exceeding the SPHERE standard of $15 \%$ and missing data. Each of these limitations may impact the internal and external validity of our results. However, the analysis uniquely presents data from a large programmatic cohort from a context with a high burden of SAM to provide further insight into the key implications of using MUAC as a sole anthropomorphic admission criterion in terms of treatment response, recovery, transfer and death.

\section{Conclusion}

In the present retrospective study we found that children who would have been admitted to an out-patient TFP using a single MUAC $<115 \mathrm{~mm}$ criterion were more likely to be more severely malnourished, female and of younger age than children who would have been excluded if a single MUAC $<115 \mathrm{~mm}$ criterion were adopted. Children who would have been admitted using a single MUAC $<115 \mathrm{~mm}$ criterion had higher mortality, but responded well to treatment, as demonstrated by greater weight and MUAC gain. In this setting, we have shown that MUAC $<115 \mathrm{~mm}$ as a single admission criterion for community-based treatment programmes for SAM would have failed to include one-third of the children who died in the programme. Our experience in the field supports continued efforts to better understand the implementation and implications of MUAC-based programming across a variety of programmatic settings, and the present analysis suggests that admission criteria will need to be adapted to the programmatic context with consideration for both operational and public health implications.

\section{Acknowledgements}

Financial support: This work was supported by MSF Operational Center Paris. Conflict of interest: None. Authorship: E.G., L.K.K., M.S.E., K.P. and S.I. conceived and designed the experiment. E.G. analysed the data. All authors contributed to the interpretation of the data and preparation of the manuscript. Ethics of buman subject participation: The data analyses were exempted from review by the MSF Ethical Review Board given the use of de-identified, routine programme data.

\section{References}

1. Black RE, Victora CG, Walker SP et al. (2013) Maternal and child undernutrition and overweight in low-income and middle-income countries. Lancet 382, 427-451.

2. Olofin I, McDonald CM, Ezzati M et al. (2013) Associations of suboptimal growth with all-cause and cause-specific mortality in children under five years: a pooled analysis of ten prospective studies. PLoS One 8, e64636.

3. United Nations System Standing Committee on Nutrition \& UNICEF (2007) Community-Based Management of Severe Acute Malnutrition. A Joint Statement by the World Health Organization, the World Food Programme, the United Nations System Standing Committee on Nutrition and the United Nations Children's Fund. http://www.who. int/nutrition/topics/Statement_community_based_man_sev_ acute_mal_eng.pdf (accessed December 2014).

4. Ciliberto MA, Sandige H, Ndekha MJ et al. (2005) Comparison of home-based therapy with ready-to-use therapeutic food with standard therapy in the treatment of malnourished Malawian children: a controlled, clinical effectiveness trial. Am J Clin Nutr 81, 864-870.

5. Lenters LM, Wazny K, Webb $\mathrm{P}$ et al. (2013) Treatment of severe and moderate acute malnutrition in lowand middle-income settings: a systematic review, metaanalysis and Delphi process. BMC Public Health 13, Suppl. 3, S23.

6. Linneman Z, Matilsky D, Ndekha M et al. (2007) A largescale operational study of home-based therapy with readyto-use therapeutic food in childhood malnutrition in Malawi. Matern Child Nutr 3, 206-215.

7. Manary MJ, Ndkeha MJ, Ashorn P et al. (2004) Home based therapy for severe malnutrition with ready-to-use food. Arch Dis Child 89, 557-561.

8. Bachmann MO (2010) Cost-effectiveness of communitybased treatment of severe acute malnutrition in children. Expert Rev Pharmacoecon Outcomes Res 10, 605-612.

9. Wilford R, Golden K \& Walker DG (2012) Cost-effectiveness of community-based management of acute malnutrition in Malawi. Health Policy Plan 27, 127-137.

10. World Health Organization (2013) Guideline updates on the management of severe acute malnutrition in infants and children. http://www.who.int/nutrition/publications/ guidelines/updates_management_SAM_infantandchildren/en/ (accessed December 2014). 
11. Berkley J, Mwangi I, Griffiths K et al. (2005) Assessment of severe malnutrition among hospitalized children in rural Kenya: comparison of weight for height and mid upper arm circumference. JAMA 294, 591-597.

12. Bern C \& Nathanail L (1995) Is mid-upper-arm circumference a useful tool for screening in emergency settings? Lancet 345, 631-633.

13. Briend A, Maire B, Fontaine O et al. (2012) Mid-upper arm circumference and weight-for-height to identify high-risk malnourished under-five children. Matern Child Nutr 8, 130-133.

14. Ali E, Zachariah R, Shams Z et al. (2013) Is mid-upper arm circumference alone sufficient for deciding admission to a nutritional programme for childhood severe acute malnutrition in Bangladesh? Trans R Soc Trop Med Hyg 107, 319-323.

15. Harvey P \& Rogers-Witte B (2007) Nutrition Status and its Determinants in Southern Sudan: A Summary of Available Data. Washington, DC: Academy for Educational Development.

16. Ruff CB \& Walker A (1993) Body size and body shape. In The Nariokotome Homo erectus Skeleton, pp. 234-265 [A Walker and REF Leakey, editors]. Cambridge, MA: Harvard University Press.

17. Myatt M, Duffield A, Seal A et al. (2009) The effect of body shape on weight-for-height and mid-upper arm circumference based case definitions of acute malnutrition in Ethiopian children. Ann Hum Biol 36, 5-20.

18. WHO Multicentre Growth Reference Study Group (2009) WHO Child Growth Standards: Methods and Development. Growth Velocity Based on Weight, Length and Head Circumference. Geneva: WHO.

19. R Development Core Team (2004) R: a Language and Environment for Statistical Computing. Vienna: R Foundation for Statistical Computing.

20. Goossens S, Bekele Y, Yun O et al. (2012) Mid-upper arm circumference based nutrition programming: evidence for a new approach in regions with high burden of acute malnutrition. PLoS One 7, e49320.

21. Myatt M, Khara T \& Collins S (2006) A review of methods to detect cases of severely malnourished children in the community for their admission into community-based therapeutic care programs. Food Nutr Bull 27, 3 Suppl., S7-S23.

22. Defourny I, Minetti A, Harczi G et al. (2009) A large-scale distribution of milk-based fortified spreads: evidence for a new approach in regions with high burden of acute malnutrition. PLoS One 4, e5455.

23. Nielsen J, Valentiner-Branth P, Martins C et al. (2004) Malnourished children and supplementary feeding during the war emergency in Guinea-Bissau in 1998-1999. Am J Clin Nutr 80, 1036-1042.

24. Fernandez MA, Delchevalerie P \& Van Herp M (2010) Accuracy of MUAC in the detection of severe wasting with the new WHO growth standards. Pediatrics 126, e195-e201.

25. Laillou A, Prak S, de Groot R et al. (2014) Optimal screening of children with acute malnutrition requires a change in current WHO guidelines as MUAC and WHZ identify different patient groups. PLoS One 9, e101159.

26. Roberfroid D, Hammami N, Lachat C et al. (2013) Utilization of a Mid-Upper Arm Circumference Versus Weight-for-Height in Nutritional Rehabilitation Programmes: A Systematic Review of Evidence. Geneva: WHO.

27. Pelletier DL (1994) The relationship between child anthropometry and mortality in developing countries: implications for policy, programs and future research. J Nutr 124, 10 Suppl., 2047S-2081S

28. Lapidus N, Luquero FJ, Gaboulaud V et al. (2009) Prognostic accuracy of WHO growth standards to predict mortality in a large-scale nutritional program in Niger. PLoS Med 6, e39.

29. Binns P, Dale NM, Banda T et al. (2014) Safety of Using Mid-Upper Arm Circumference as a Discharge Criterion in Community-Based Management of Severe Acute Malnutrition in Children Aged 6-59 Months. Oxford: Valid International.

30. Dale NM, Myatt M, Prudhon C et al. (2013) Using midupper arm circumference to end treatment of severe acute malnutrition leads to higher weight gains in the most malnourished children. PLoS One 8, e55404. 